Article

www.mdpi.com/journal/molecules

\title{
Synthesis of Some New Mono- and Bis-Polycyclic Aromatic Spiro and Bis-Nonspiro- $\beta$-Lactams
}

\author{
Aliasghar Jarrahpour * and Edris Ebrahimi \\ Department of Chemistry, College of Sciences, Shiraz University, Shiraz, 71454, Iran; \\ E-Mail: edris.ebrahimi@gmail.com (E.E.) \\ * Author to whom correspondence should be addressed; E-Mail: aliasghar6683@yahoo.com or \\ jarrah@susc.ac.ir; Tel.: +98-711-228-4822; Fax: +98-711-228-0926.
}

Received: 2 November 2009; in revised form: 3 December 2009 / Accepted: 9 December 2009 / Published: 22 January 2010

\begin{abstract}
Some new mono-and bis-polycyclic aromatic spiro- $\beta$-lactams and bis-non spiropolycyclic aromatic $\beta$-lactams have been synthesized from imines derived from anthracene-9-carbaldehyde, 2-naphtaldehyde and a ketene derived from 9H-xanthene-9carboxylic acid and phenoxyacetic acid by a [2+2] cycloaddition reaction. The cycloadducts were characterized by spectral data, including ${ }^{1} \mathrm{H}-\mathrm{NMR},{ }^{13} \mathrm{C}-\mathrm{NMR}$, IR and elemental analyses. The configurations of some of these mono-spiro- $\beta$-lactams were established by X-ray crystal analysis.
\end{abstract}

Keywords: mono- and bis-spiro- $\beta$-lactam; polycyclic aromatic imine; $9 \mathrm{H}$-xanthene-9carboxylic acid; phenoxyacetic acid; [2+2] cycloaddition; tosyl chloride

\section{Introduction}

$\beta$-Lactams, being a structural unit found in the most widely used antibiotics [1], have occupied a basic position in medicinal chemistry for almost a century now. With the microbes responding to the traditional antibiotics through $\beta$-lactamases, the need for novel antibiotics prevails, making synthesis of newer $\beta$-lactams ever more important. In addition to their use as antibiotics, $\beta$-lactams are increasingly being used as synthons for other biologically important molecules [2-11]. $\beta$-Lactams have been found to act as cholesterol acyl transferase inhibitors [12], thrombin inhibitors [13], human cytomegalovirus protease inhibitors [14], matrix metalloprotease inhibitors [15], cysteine protease [16], and apoptosis inductors [17]. Spirocyclic $\beta$-lactams have attracted attention as they have been shown 
to be $\beta$-turn mimetics [18-19] and precursors for $\alpha, \alpha$-disubstituted $\beta$-amino acids [20]. The chartelline has a spiro- $\beta$-lactam moiety in its structure marine natural products [21]. It has been found that spiro- $\beta$-lactams act as poliovirus and human rhinovirus 3 C-proteinases inhibitors [22]. These compounds are mostly synthesized by cycloaddition to an exocyclic bond. Several syntheses of spiro- $\beta$-lactams have been reported [23-42]. Polycyclic aromatic $\beta$-lactams have shown anticancer and other biological activities [43-45]. Some polyaromatic $\beta$-lactams have been reported to have polyaromatic ring in part of imines that prepared by Staudinger reaction [46]. Therefore in continuation of our work on the synthesis of novel $\beta$-lactams [47-49], we present here for the first time the results obtained in the synthesis of mono- and bis-polyaromatic spiro and nonspiro- $\beta$-lactams using a modified Staudinger reaction.

\section{Results and Discussion}

Treatment of anthracene-9-carbaldehyde or 2-naphtaldehyde with different primary amines in refluxing ethanol afforded the polycyclic aromatic imines 1a-n [50-53]. These Schiff bases were then treated with $9 H$-xanthene-9-carboxylic acid in the presence of triethylamine and tosyl chloride to afford polycyclic aromatic spiro- $\beta$-lactams $\mathbf{2 a}-\mathbf{n}$ as single diastereomers (Scheme 1). The reaction progress was monitored by TLC and the presence of a new compound was confirmed. In addition, the cycloadducts were characterized by spectral analysis. For 2a the IR spectrum showed the characteristic absorption of a $\beta$-lactam carbonyl at $1,747 \mathrm{~cm}^{-1}$. The ${ }^{1} \mathrm{H}-\mathrm{NMR}$ spectrum exhibited the methoxy protons as a singlet at $3.64 \mathrm{ppm}$, the $\beta$-lactam $\mathrm{H}-4$ proton as a singlet at 6.29 and aromatic protons as a multiplet at 6.39-8.82. The ${ }^{13} \mathrm{C}-\mathrm{NMR}$ spectrum exhibited the C-3 (spiro carbon) at 64.3 . The results for other polycyclic aromatic spiro- $\beta$-lactams $\mathbf{2} \mathbf{a}-\mathbf{n}$ are shown in Table 1.

Scheme 1. Synthesis of polycyclic aromatic spiro- $\beta$-lactams $\mathbf{2 a}-\mathbf{n}$.

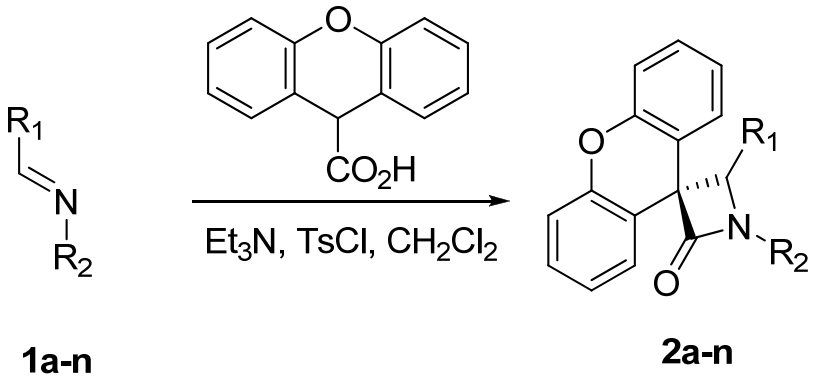<smiles>[R]=[Pt]=Cc1ccc2cc3ccccc3c(C)c2c1</smiles> 
Table 1. Structures of spiro- $\beta$-lactams.

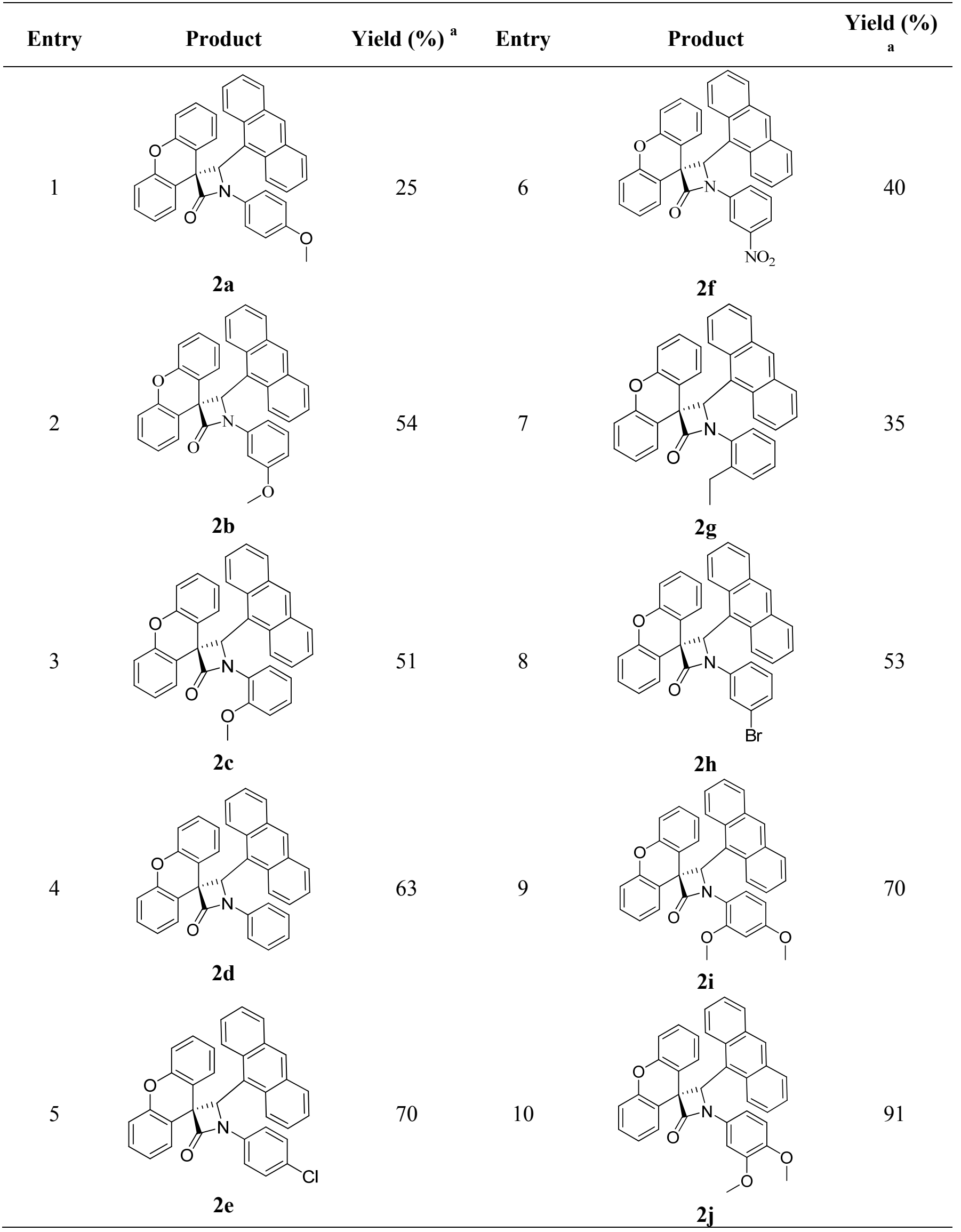


Table 1. Cont.

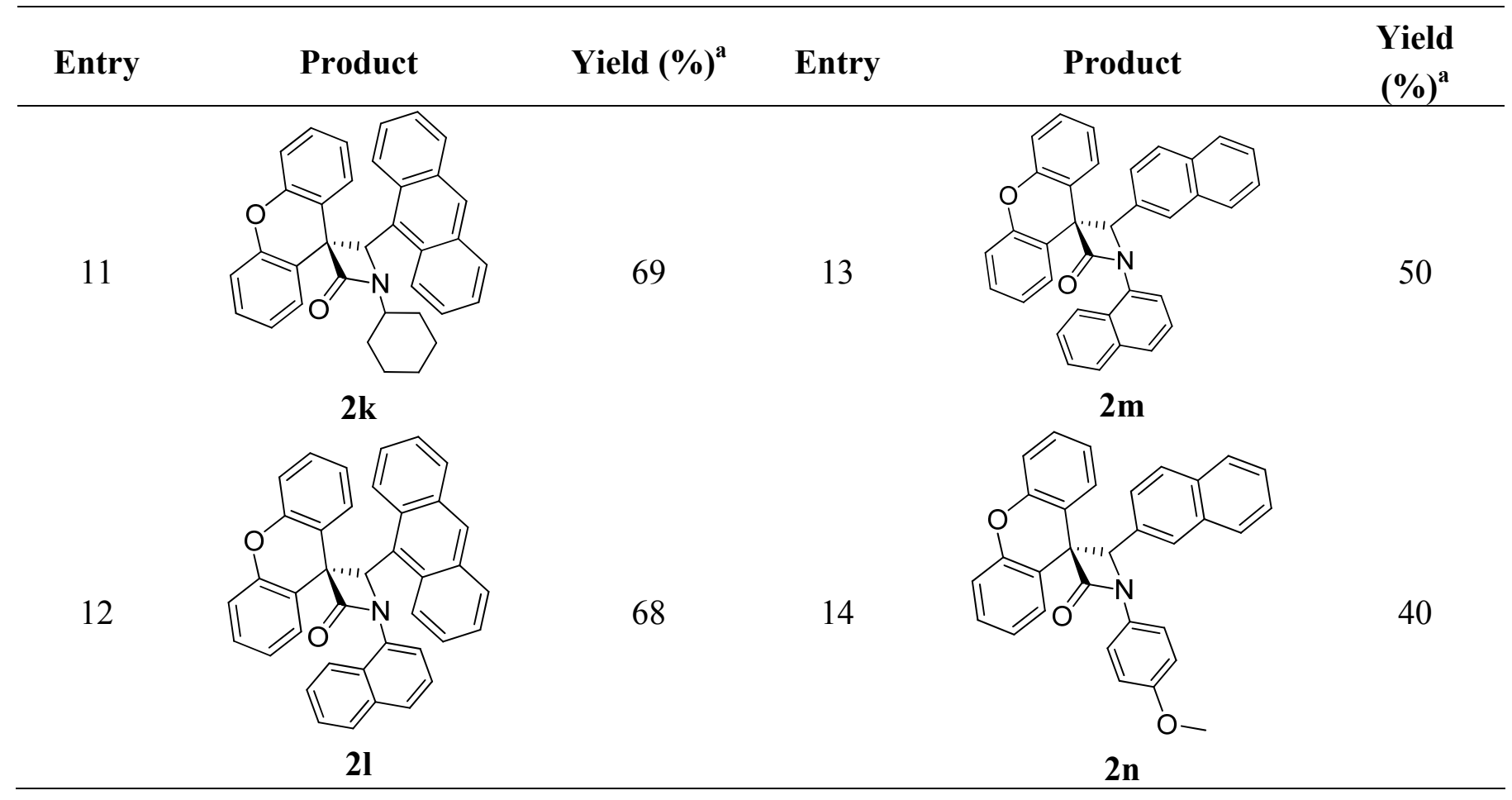

${ }^{\mathrm{a}}$ Isolated yield of pure products.

Then we decided to synthesize bis-spiro- and bis-nonspiro- polycyclic aromatic $\beta$-lactams $\mathbf{4 a - d}, \mathbf{6 b}$ and $\mathbf{4 e - h}, \mathbf{6 a}$ from bis-imines $\mathbf{3 a}-\mathbf{d}$ and $\mathbf{5}$ [53] (Scheme 2). The structures of $\mathbf{4 a}-\mathbf{h}$ and $\mathbf{5 a}-\mathbf{b}$ are shown in Table 2. The cis-trans stereochemistry of 2-azetidinones $4 \mathbf{e}-\mathbf{h}$ and $\mathbf{6 a}$ were deduced from the coupling constant of $\mathrm{H}-3$ and H-4, which was calculated to be $J_{3,4}=4-9 \mathrm{~Hz}$ for the cis and $J_{3,4}=1-3 \mathrm{~Hz}$ for the trans stereoisomers.

Scheme 2. Synthesis of bis-spiro- and bis-nonspiro- polycyclic aromatic $\beta$-lactams $4 \mathbf{a}-\mathbf{h}$ and $\mathbf{6 a - b}$.

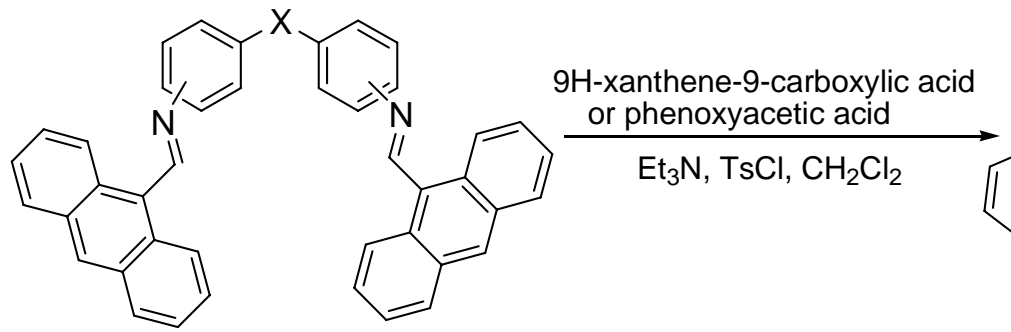

3a: $\mathrm{X}=\mathrm{CH}_{2}\left(4,4^{\prime}\right)$

3b: $X=\mathrm{CH}_{2}\left(3,3^{\prime}\right)$

3c: $X=O \quad\left(4,4^{\prime}\right)$

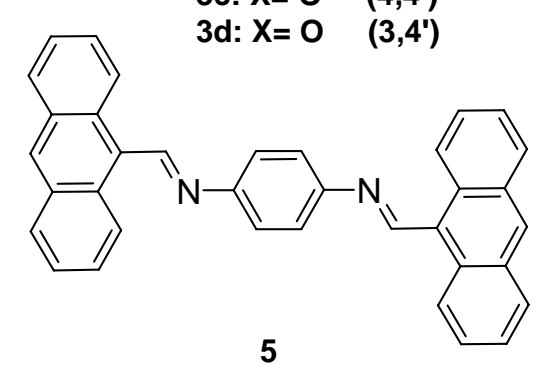

9H-xanthene-9-carboxylic acid or phenoxyacetic acid<smiles>[X]c1ccc(N2C(=O)C([R2])C2c2c3ccccc3cc3ccccc23)cc1</smiles>

4a-h

$\mathrm{Et}_{3} \mathrm{~N}, \mathrm{TsCl}, \mathrm{CH}_{2} \mathrm{Cl}_{2}$<smiles></smiles> 
Table 2. Structures of bis-spiro and bis-nonspiro- $\beta$-lactams.

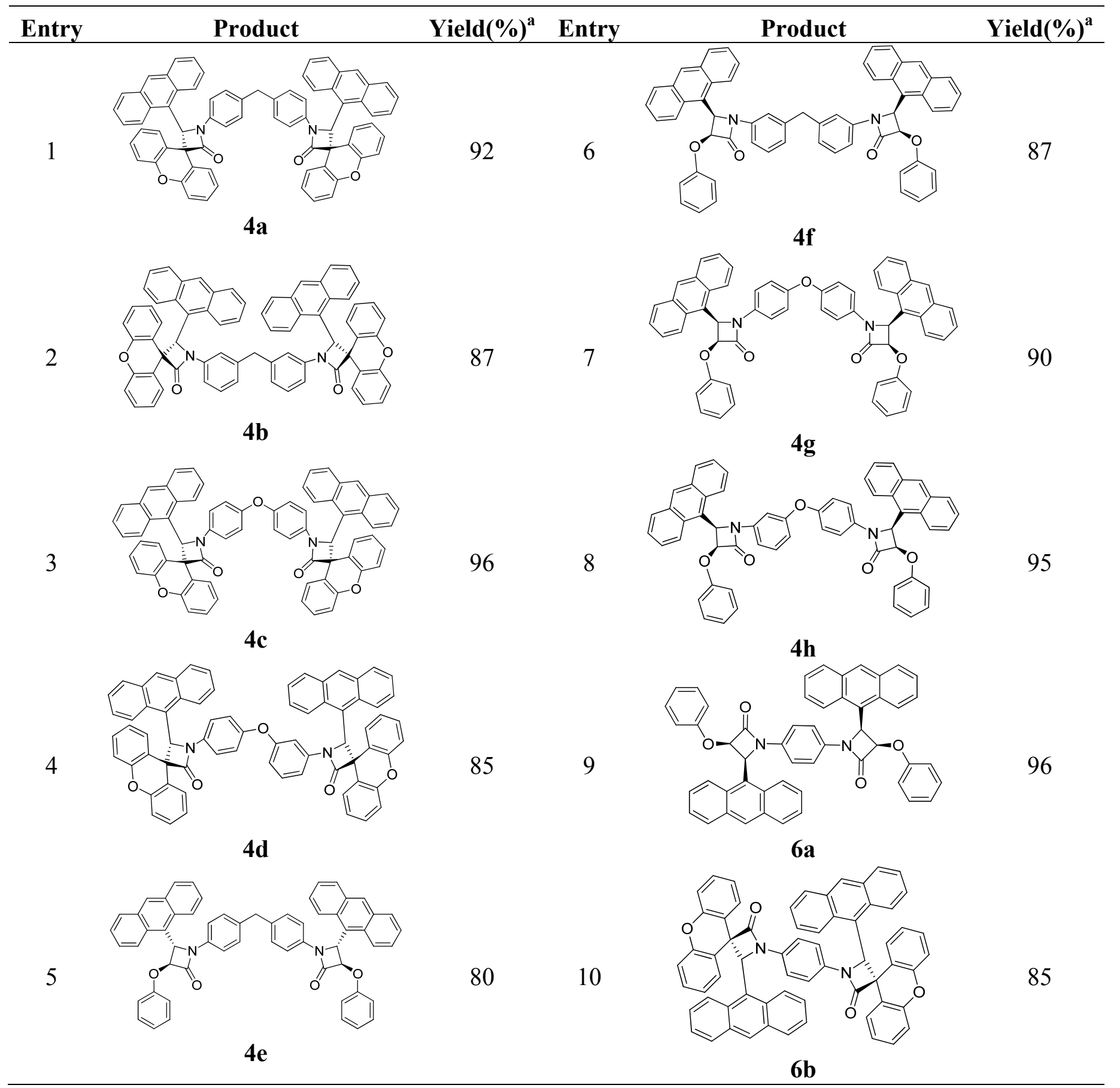

${ }^{\mathrm{a}}$ Isolated yields of pure products.

The X-ray crystallography of $\mathbf{2 a}$ (Figure 1) confirmed the proposed spiro configuration at C3 [54-59]. The X-ray analysis also showed that the $\beta$-lactam ring is planar and it is perpendicular to xanthene ring. The anthracene ring has dihedral angles of $51.8^{\circ}$ with the $\beta$-lactam ring. 
Figure 1. X-ray crystal structure of $\mathbf{2 a}$.

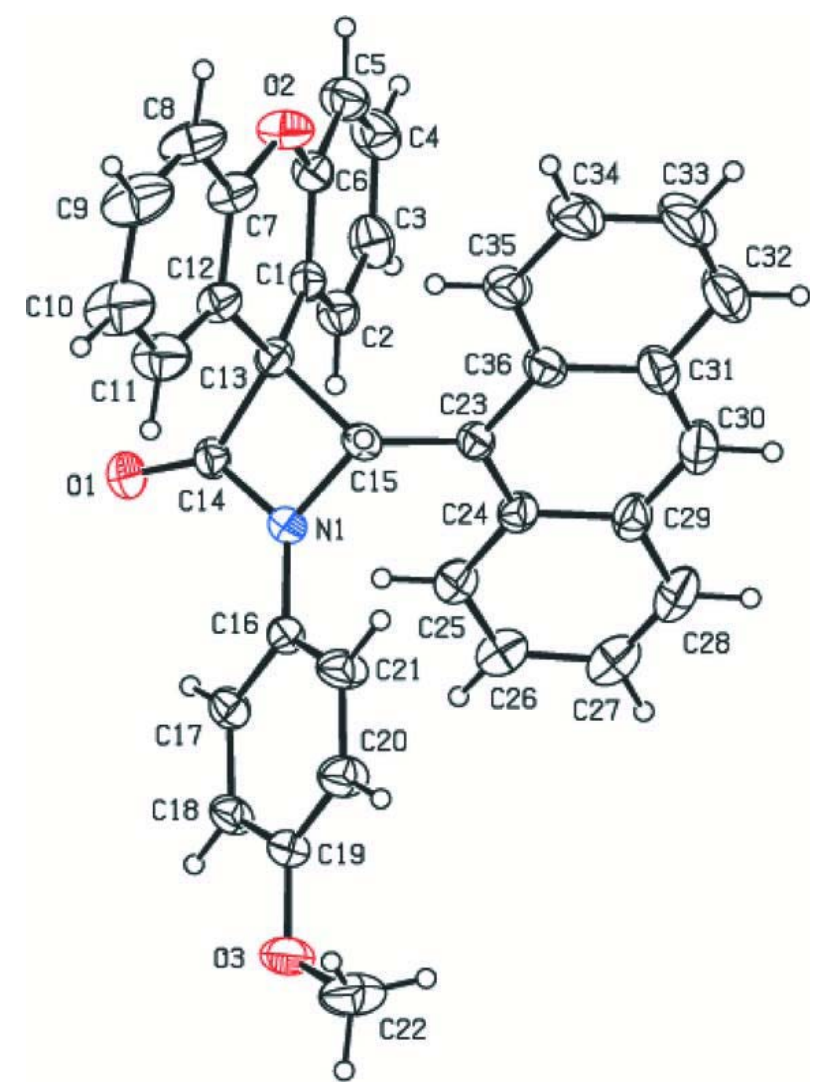

\section{Experimental Section}

\subsection{General}

All needed chemicals were purchased from the Merck, Fluka or Acros chemical companies. All reagents and solvents were dried prior to use according to standard methods [60]. IR spectra were run on a Shimadzu FT-IR 8300 spectrophotometer. ${ }^{1} \mathrm{H}-\mathrm{NMR}$ and ${ }^{13} \mathrm{C}-\mathrm{NMR}$ spectra were recorded in DMSO-d $\mathrm{d}_{6}$ or $\mathrm{CDCl}_{3}$ using a Bruker Avance DPX instrument $\left({ }^{1} \mathrm{H}-\mathrm{NMR} 250 \mathrm{MHz},{ }^{13} \mathrm{C}-\mathrm{NMR} 62.9 \mathrm{MHz}\right)$. Chemical shifts were reported in parts per million $(\delta)$ downfield from TMS. All of the coupling constants $(J)$ are in hertz. The mass spectra were recorded on a Shimadzu GC-MS QP 1000 EX instrument. Elemental analyses were run on a Thermo Finnigan Flash EA-1112 series. Melting points were determined in open capillaries with Buchi 510 melting point apparatus. Thin-layer chromatography was carried out on silica gel F254 analytical sheets obtained from Fluka. Column chromatography was performed on Merck Kiesel gel (230-270 mesh).

\subsection{General Procedure for Preparation of Schiff Bases 1a-n}

A mixture of an aromatic amine $(1.00 \mathrm{mmol})$ and anthracene-9-carbaldehyde or 2-naphtaldehyde (1.00 mmol) was refluxed in ethanol $(20 \mathrm{~mL})$ for $1-5$ hours. After cooling, the pure Schiff bases were separated as crystals. Some of these were recrystallized from ethanol.

(E)-N-(Anthracen-10-ylmethlene)-4-methxybenzenamine (1a): Yellow powder crystal (yield 91\%); Mp: $140-148{ }^{\circ} \mathrm{C}$; IR $\left(\mathrm{CHCl}_{3}, \mathrm{~cm}^{-1}\right): 1,608(\mathrm{C}=\mathrm{N}) ;{ }^{1} \mathrm{H}-\mathrm{NMR} \delta(\mathrm{ppm}): 3.88(\mathrm{OMe}, \mathrm{s}, 3 \mathrm{H}), 6.52-7.64$ 
(ArH, m, 13H), $9.80(\mathrm{CHN}, \mathrm{s}, 1 \mathrm{H})$. Analysis calculated for $\mathrm{C}_{22} \mathrm{H}_{17} \mathrm{NO}: \mathrm{C}, 84.86 ; \mathrm{H}, 5.50 ; \mathrm{N}, 4.50 \%$. Found: C, 84.83; H, 5.55; N, 4.48\%.

(E)-N-(Anthracen-10-ylmethylene)-3-methoxybenzenamine (1b): Yellow powder crystal (yield 84\%); Mp: $100-102{ }^{\circ} \mathrm{C}$; IR $\left(\mathrm{CHCl}_{3}, \mathrm{~cm}^{-1}\right): 1,608(\mathrm{C}=\mathrm{N}) ;{ }^{1} \mathrm{H}-\mathrm{NMR} \delta(\mathrm{ppm}): 3.87(\mathrm{OMe}, \mathrm{s}, 3 \mathrm{H}), 6.89-8.74$ $(\mathrm{ArH}, \mathrm{m}, 13 \mathrm{H}), 9.66(\mathrm{CHN}, \mathrm{s}, 1 \mathrm{H})$. Analysis calculated for $\mathrm{C}_{22} \mathrm{H}_{17} \mathrm{NO}: \mathrm{C}, 84.86 ; \mathrm{H}, 5.50 ; \mathrm{N}, 4.50 \%$. Found: C, 84.83; H, 5.55; N, 4.48\%.

(E)-N-(Anthracen-10-ylmethylene)-2-methoxybenzenamine (1c): Orange powder crystal (yield 76\%); Mp: 94-96 ${ }^{\circ} \mathrm{C}$; IR $\left(\mathrm{CHCl}_{3}, \mathrm{~cm}^{-1}\right): 1,620(\mathrm{C}=\mathrm{N}) ;{ }^{1} \mathrm{H}-\mathrm{NMR} \delta(\mathrm{ppm}): 6.73-8.75(\mathrm{ArH}, \mathrm{m}, 13 \mathrm{H}), 9.66$ $(\mathrm{CHN}, \mathrm{s}, 1 \mathrm{H})$. Analysis calculated for $\mathrm{C}_{22} \mathrm{H}_{17} \mathrm{NO}$ : C, 84.86; H, 5.50; N, 4.50\%. Found: C, 84.83; H, $5.55 ; \mathrm{N}, 4.48 \%$.

(E)-N-(Anthracen-10-ylmethylene)benzenamine (1d): Yellow powder crystal (yield 76\%); Mp: $102-104{ }^{\circ} \mathrm{C}$; IR ( $\left.\mathrm{KBr}, \mathrm{cm}^{-1}\right): 1,608(\mathrm{C}=\mathrm{N}) ;{ }^{1} \mathrm{H}-\mathrm{NMR} \delta(\mathrm{ppm}): 6.61-8.96(\mathrm{ArH}, \mathrm{m}, 14 \mathrm{H}), 9.63$ $(\mathrm{CHN}, \mathrm{s}, 1 \mathrm{H})$. Analysis calculated for $\mathrm{C}_{21} \mathrm{H}_{15} \mathrm{~N}$ : C, 89.65; H, 5.37; N, 4.98\%. Found: C, 89.61; H, $5.41 ; \mathrm{N}, 4.95 \%$.

(E)-N-(Anthracen-10-ylmethylene)-4-chlorobenzenamine (1e): Yllow crystal (yield 51\%); Mp: $182-184{ }^{\circ} \mathrm{C}$; IR (KBr, cm $\left.{ }^{-1}\right): 1,623(\mathrm{C}=\mathrm{N}) ;{ }^{1} \mathrm{H}-\mathrm{NMR} \delta(\mathrm{ppm}): 6.64-8.72(\mathrm{ArH}, \mathrm{m}, 13 \mathrm{H}), 9.61$ $(\mathrm{CHN}, \mathrm{s}, 1 \mathrm{H})$. Analysis calculated for $\mathrm{C}_{21} \mathrm{H}_{14} \mathrm{ClN}$ : C, 79.87; H, 4.47; N, 4.44\%. Found: C, 79.85; H, 4.50; N, 4.49\%.

(E)-N-(Anthracen-10-ylmethylene)-3-nitrobenzenamine (1f): Orange crystal (yield 67\%); Mp: $166-168{ }^{\circ} \mathrm{C}$; IR $\left(\mathrm{KBr}, \mathrm{cm}^{-1}\right): 1,666(\mathrm{C}=\mathrm{N})$; ${ }^{1} \mathrm{H}-\mathrm{NMR} \delta(\mathrm{ppm}): 6.62-8.96(\mathrm{ArH}, \mathrm{m}, 13 \mathrm{H}), 9.66$ $(\mathrm{CHN}, \mathrm{s}, 1 \mathrm{H})$. Analysis calculated for $\mathrm{C}_{21} \mathrm{H}_{14} \mathrm{~N}_{2} \mathrm{O}_{2}$ : C, 77.29; H, 4.32; N, 8.58\%. Found: C, 77.35; $\mathrm{H}, 4.38 ; \mathrm{N}, 8.60 \%$.

(E)-N-(Anthracen-10-ylmethylene)-2-ethylbenzenamine (1g): Yellow crystal (yield 60\%); Mp: $182-184{ }^{\circ} \mathrm{C}$; IR $\left(\mathrm{CHCl}_{3}, \mathrm{~cm}^{-1}\right): 1,620(\mathrm{C}=\mathrm{N}) ;{ }^{1} \mathrm{H}-\mathrm{NMR} \delta(\mathrm{ppm}): 1.27\left(\mathrm{CH}_{2}, \mathrm{t}, 2 \mathrm{H}, J=7.5\right), 2.89$ $\left(\mathrm{CH}_{3}, \mathrm{q}, 4 \mathrm{H}, J=7.5\right), 6.82-8.80(\mathrm{ArH}, \mathrm{m}, 13 \mathrm{H}), 9.55(\mathrm{CHN}, \mathrm{s}, 1 \mathrm{H})$. Analysis calculated for $\mathrm{C}_{23} \mathrm{H}_{19} \mathrm{~N}$ : C, 89.28; H, 6.19; N, 4.53\%. Found: C, 89.32; H, 6.25; N, 4.55\%.

(E)-N-(Anthracen-10-ylmethylene)-3-bromobenzenamine (1h): Yellow crystal (yield 77\%); Mp: $122-124{ }^{\circ} \mathrm{C}$; IR $\left(\mathrm{KBr}, \mathrm{cm}^{-1}\right): 1,620(\mathrm{C}=\mathrm{N})$; ${ }^{1} \mathrm{H}-\mathrm{NMR} \delta(\mathrm{ppm}): 6.84-8.71(\mathrm{ArH}, \mathrm{m}, 13 \mathrm{H}), 9.60$ $(\mathrm{CHN}, \mathrm{s}, 1 \mathrm{H})$. Analysis calculated for $\mathrm{C}_{21} \mathrm{H}_{14} \mathrm{BrN}$ : C, 70.01; H, 3.92; N, 3.89\%. Found: C, 70.11; H, 3.98; N, 3.84\%.

(E)-N-(Anthracen-10-ylmethylene)-2,4-dimethoxybenzenamine (1i): Orange crystal (yield 88\%);

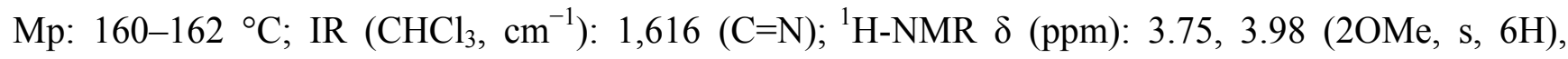
6.51-8.73 (ArH, m, 12H), $9.92(\mathrm{CHN}, \mathrm{s}, 1 \mathrm{H})$. Analysis calculated for $\mathrm{C}_{23} \mathrm{H}_{19} \mathrm{NO}_{2}$ : C, 80.92; H, 5.61; N, 4.10\%. Found: C, 80.96; H, 5.66; N, 4.17\%. 
(E)-N-(Anthracen-10-ylmethylene)-3,4-dimethoxybenzenamine (1j): Orange crystal (yield 90\%); Mp: $160-162{ }^{\circ} \mathrm{C}$; IR $\left(\mathrm{CHCl}_{3}, \mathrm{~cm}^{-1}\right): 1,605(\mathrm{C}=\mathrm{N}) ;{ }^{1} \mathrm{H}-\mathrm{NMR} \delta(\mathrm{ppm}): 3.88,3.91$ (2OMe, s, 6H), 6.88-8.68 (ArH, m, 12H), $9.92(\mathrm{CHN}, \mathrm{s}, 1 \mathrm{H})$. Analysis calculated for $\mathrm{C}_{23} \mathrm{H}_{19} \mathrm{NO}_{2}$ : C, 80.92; H, 5.61; N, 4.10\%. Found: C, 80.96; H, 5.66; N, 4.17\%.

(E)-N-(Anthracen-10-ylmethylene)cyclohexanamine (1k): Orange crystal (yield 92\%); Mp: $132-134{ }^{\circ} \mathrm{C}$; IR $\left(\mathrm{CHCl}_{3}, \mathrm{~cm}^{-1}\right): 1,639(\mathrm{C}=\mathrm{N}) ;{ }^{1} \mathrm{H}-\mathrm{NMR} \delta(\mathrm{ppm}): 1.34-3.56$ (cyclohexyl, m, 11H), 7.41-8.43 (ArH, m, 9H), $9.34(\mathrm{CHN}, \mathrm{s}, 1 \mathrm{H})$. Analysis calculated for $\mathrm{C}_{21} \mathrm{H}_{21} \mathrm{~N}: \mathrm{C}, 87.76 ; \mathrm{H}, 7.36 ; \mathrm{N}$, 4.87\%. Found: C, 87.71; H, 7.33; N, 4.91\%.

(E)-N-(Anthracen-10-ylmethlene)naphthalene-1-amine (11): Yellow crystal (yield 60\%); Mp: $142-144^{\circ} \mathrm{C}$; IR $\left(\mathrm{CHCl}_{3}, \mathrm{~cm}^{-1}\right): 1,624(\mathrm{C}=\mathrm{N}) ;{ }^{1} \mathrm{H}-\mathrm{NMR} \delta(\mathrm{ppm}): 6.67-8.94(\mathrm{Ar}-\mathrm{H}, \mathrm{m}, 16 \mathrm{H}), 8.80$ $(\mathrm{CHN}, \mathrm{s}, 1 \mathrm{H})$. Analysis calculated for $\mathrm{C}_{25} \mathrm{H}_{17} \mathrm{~N}$ : C, 90.60; H, 5.17; N, 4.23\%. Found: C, 90.66; $\mathrm{H}, 5.20 ; \mathrm{N}, 4.28 \%$.

(E)-N-(Naphthalen-2-ylmethylene)naphthalen-1-amine (1m): Green crystal (yield 78\%); Mp: $129-130^{\circ} \mathrm{C}$. IR $\left(\mathrm{CHCl}_{3}, \mathrm{~cm}^{-1}\right): 1,624(\mathrm{C}=\mathrm{N}) ;{ }^{1} \mathrm{H}-\mathrm{NMR} \delta(\mathrm{ppm}): 6.73-8.63(\mathrm{Ar}-\mathrm{H}, \mathrm{m}, 14 \mathrm{H}), 8.94$ $(\mathrm{CHN}, \mathrm{s}, 1 \mathrm{H})$. Analysis calculated for $\mathrm{C}_{25} \mathrm{H}_{17} \mathrm{~N}$ : C, 90.60; H, 5.17; N, 4.23\%. Found: C, 90.66; H, 5.20; $\mathrm{N}, 4.28 \%$.

(E)-4-Methoxy-N-(naphthalen-2-ylmethylene)benzenamine (1n): Silver powder crystal (yield 80\%); Mp: $118-120^{\circ} \mathrm{C}$; IR $\left(\mathrm{CHCl}_{3}, \mathrm{~cm}^{-1}\right): 1,620(\mathrm{C}=\mathrm{N}) ;{ }^{1} \mathrm{H}-\mathrm{NMR} \delta(\mathrm{ppm}): 3.78(\mathrm{OMe}, \mathrm{s}, 3 \mathrm{H}), 6.90-8.13$ $(\mathrm{ArH}, \mathrm{m}, 11 \mathrm{H}), 8.60(\mathrm{CHN}, \mathrm{s}, 1 \mathrm{H})$. Analysis calculated for $\mathrm{C}_{18} \mathrm{H}_{15} \mathrm{NO}: \mathrm{C}, 82.73 ; \mathrm{H}, 5.79 ; \mathrm{N}, 5.36 \%$. Found: C, 82.75; H, 5.83; N, 5.32\%.

\subsection{General Procedure for Preparation of Bis-Schiff Bases 3a-d and $\mathbf{5}$}

A mixture of anthracene-9-carbaldehyde $(1.00 \mathrm{mmol})$ and bisamine $(0.50 \mathrm{mmol})$ was refluxed in ethanol $(20 \mathrm{~mL})$ for $(1-5) \mathrm{h}$. After cooling, the pure Schiff bases were separated as crystals. Some of these were recrystallized from ethanol.

(E)-4-(E)-4-[(E)-Anthracen-10-ylmethyleneamino)benzyl]-N-(anthracen-10-ylmethylene)benzeneamine (3a): Yellow powder (yield 96\%); Mp: 216-218 ${ }^{\circ} \mathrm{C}$; IR $\left(\mathrm{KBr}, \mathrm{cm}^{-1}\right): 1,623(\mathrm{C}=\mathrm{N}) ;{ }^{1} \mathrm{H}-\mathrm{NMR}$ $\delta$ (ppm): $4.15\left(\mathrm{CH}_{2}, \mathrm{~s}, 2 \mathrm{H}\right), 6.68-9.70(\mathrm{ArH}, \mathrm{m}, 26 \mathrm{H}), 10.02(\mathrm{CH}=\mathrm{N}, \mathrm{s}, 2 \mathrm{H})$; Analysis calculated for $\mathrm{C}_{43} \mathrm{H}_{30} \mathrm{~N}_{2}$ : C, 89.86; H, 5.26; N, 4.87\%. Found: C, 89.81; H, 5.22; N, 4.83\%.

(E)-3-(E)-3-[(E)-Anthracen-10-ylmethyleneamino)benzyl]-N-(anthracen-10-yl-methylene)benzenenamine (3b): Yellow powder (yield 95\%); Mp: $184-186{ }^{\circ} \mathrm{C}$; IR $\left(\mathrm{KBr}, \mathrm{cm}^{-1}\right): 1,623(\mathrm{C}=\mathrm{N}) ;{ }^{1} \mathrm{H}-\mathrm{NMR} \delta(\mathrm{ppm})$ : $4.19\left(\mathrm{CH}_{2}, \mathrm{~s}, 2 \mathrm{H}\right), 7.24-8.92(\mathrm{ArH}, \mathrm{m}, 26 \mathrm{H}), 9.73(\mathrm{CH}=\mathrm{N}, \mathrm{s}, 2 \mathrm{H})$; Analysis calculated for $\mathrm{C}_{43} \mathrm{H}_{30} \mathrm{~N}_{2}$ : C, 89.86; H, 5.26; N, 4.87\%. Found: C, 89.81; H, 5.22; N, 4.83\%. 
(E)-N-(Anthracen-10-yl-methylene)-4-(4-[(E)-anthracen-10-ylmethyleneamino]phenoxy)benzeneamine (3c): Orange powder (yield 96\%); Mp: 202-204 ${ }^{\circ} \mathrm{C}$; IR $\left(\mathrm{KBr}, \mathrm{cm}^{-1}\right): 1,608(\mathrm{C}=\mathrm{N}) ;{ }^{1} \mathrm{H}-\mathrm{NMR} \delta$ (ppm): 6.73-8.78 (ArH, m, 26H), $9.60(\mathrm{CH}=\mathrm{N}, \mathrm{s}, 2 \mathrm{H})$; Analysis calculated for $\mathrm{C}_{42} \mathrm{H}_{28} \mathrm{~N}_{2} \mathrm{O}: \mathrm{C}, 87.47$; $\mathrm{H}, 4.89$; N, 4.86\%. Found: C, 87.51; H, 4.95; N, 4.81\%.

(E)-N-(Anthracen-10-ylmethylene)-3-(4-[(E)-anthracen-10-ylmethyleneamino]phenoxy)benzeneamine (3d): Yellow powder (yield 88\%); Mp: $216-218^{\circ} \mathrm{C}$; IR $\left(\mathrm{KBr}, \mathrm{cm}^{-1}\right): 1,620(\mathrm{C}=\mathrm{N})$; ${ }^{1} \mathrm{H}-\mathrm{NMR} \delta(\mathrm{ppm})$ : 6.20-9.01 (ArH, m, 26H), $9.71(\mathrm{CH}=\mathrm{N}, \mathrm{s}, 2 \mathrm{H})$; Analysis calculated for $\mathrm{C}_{42} \mathrm{H}_{28} \mathrm{~N}_{2} \mathrm{O}$ : C, 87.47; H, 4.89; N, 4.86\%. Found: C, 87.51; H, 4.95; N, 4.81\%.

$\left(N^{l} E, N^{4} E\right)-N^{l}, N^{4}$-bis(Anthracen-10-ylmethylene)benzene-1,4-diamine (5): Yellow powder (yield 95\%); Mp: $>240{ }^{\circ} \mathrm{C}$; IR $\left(\mathrm{KBr}, \mathrm{cm}^{-1}\right): 1,604(\mathrm{C}=\mathrm{N}) ;{ }^{1} \mathrm{H}-\mathrm{NMR} \delta(\mathrm{ppm}): 7.25-8.84(\mathrm{ArH}, \mathrm{m}, 22 \mathrm{H}), 9.81(\mathrm{CHN}$, s, 2H). Analysis calculated for $\mathrm{C}_{36} \mathrm{H}_{24} \mathrm{~N}_{2}$ : C, 89.23; H, 4.99; N, 5.78\%. Found: C, 89.28; H, 5.05; $\mathrm{N}, 5.73 \%$.

\subsection{General Procedure for the Synthesis of Polycyclic Aromatic Spiro- $\beta$-Lactams 2a-n}

A mixture of Schiff base $(1.00 \mathrm{mmol})$, triethylamine $(5.00 \mathrm{mmol}), 9 H$-xanthene-9-carboxylic acid $(1.50 \mathrm{mmol})$ and tosyl chloride $(1.50 \mathrm{mmol})$ in dry $\mathrm{CH}_{2} \mathrm{Cl}_{2}(15 \mathrm{~mL})$ was stirred at room temperature for $24 \mathrm{~h}$. Then it was washed with $\mathrm{HCl} 1 \mathrm{~N}(20 \mathrm{~mL})$, saturated $\mathrm{NaHCO}_{3}(20 \mathrm{~mL})$ and brine $(20 \mathrm{~mL})$. The organic layer was dried $\left(\mathrm{Na}_{2} \mathrm{SO}_{4}\right)$, filtered and the solvent was evaporated to give the product as a crystal which was then purified by recrystallization from appropriate organic solvents.

2-(Anthracen-9-yl)-1-(4-methoxyphenyl)spiro[azetidine-3,9'-xanthen]-4-one (2a): Light yellow crystals from EtOAc (yield 25\%); Mp: $223-225{ }^{\circ} \mathrm{C}$; IR $\left(\mathrm{CHCl}_{3}, \mathrm{~cm}^{-1}\right): 1,740\left(\mathrm{CO} \beta\right.$-lactam); ${ }^{1} \mathrm{H}-\mathrm{NMR}$ $\delta$ (ppm): $3.64(\mathrm{OMe}, \mathrm{s}, 3 \mathrm{H}) 6.29(\mathrm{H}-4, \mathrm{~s}, 1 \mathrm{H}), 6.51-8.82(\mathrm{ArH}, \mathrm{m}, 21 \mathrm{H}) ;{ }^{13} \mathrm{C}-\mathrm{NMR} \delta(\mathrm{ppm}) 64.3$ (OMe) 82.8 (C-3), 75.6 (C-4), 116.4-152.2 (aromatic carbon), 167.3 (CO $\beta$-lactam); GC-MS m/z = $519\left[\mathrm{M}^{+}\right]$; Analysis calculated for $\mathrm{C}_{36} \mathrm{H}_{25} \mathrm{NO}_{3}: \mathrm{C}, 83.22 ; \mathrm{H}, 4.85 ; \mathrm{N}, 2.70 \%$. Found: C, 83.95; H, 4.90; N, 2.82 .

2-(Anthracen-9-yl)-1-(3-methoxyphenyl)spiro[azetidine-3,9'-xanthen]-4-one (2b): Yellow crystals from EtOAc (yield 54\%); Mp: 212-214 ${ }^{\circ} \mathrm{C}$; IR $\left(\mathrm{CHCl}_{3}, \mathrm{~cm}^{-1}\right)$ : 1,755 (CO $\beta$-lactam); ${ }^{1} \mathrm{H}-\mathrm{NMR} \delta$ (ppm): $\quad 3.75$ (OMe, s, $\quad 3 \mathrm{H}) \quad 6.33 \quad(\mathrm{H}-4, \quad \mathrm{~s}, \quad 1 \mathrm{H}), \quad 6.53-8.80 \quad(\mathrm{ArH}, \quad \mathrm{m}, \quad 21 \mathrm{H})$; ${ }^{13} \mathrm{C}-\mathrm{NMR} \delta(\mathrm{ppm}): 55.3$ (OMe) 65.6 (C-3), 75.6 (C-4), 103.5-160.4 (aromatic carbon), 167.6 (CO $\beta$-lactam); GC-MS m/z $=519\left[\mathrm{M}^{+}\right]$; Analysis calculated for $\mathrm{C}_{36} \mathrm{H}_{25} \mathrm{NO}_{3}: \mathrm{C}, 83.22 ; \mathrm{H}, 4.85 ; \mathrm{N}, 2.70 \%$. Found: C, 83.95; H, 4.90; N, 2.82\%.

2-(Anthracen-9-yl)-1-(2-methoxyphenyl)spiro[azetidine-3,9'-xanthen]-4-one (2c): Light yellow crystals from EtOAc (yield 57\%); Mp: $214-216{ }^{\circ} \mathrm{C}$ (dec.); IR (KBr, $\mathrm{cm}^{-1}$ ): 1,739 (CO $\beta$-lactam); ${ }^{1} \mathrm{H}-\mathrm{NMR} \quad \delta \quad(\mathrm{ppm}): 2.94$ (OMe, s, 3H), 6.34 (H-4, s, 1H), 6.55-9.20 (ArH, m, 21H); ${ }^{13} \mathrm{C}-\mathrm{NMR} \delta(\mathrm{ppm}): 55.4$ (OMe), 66.0 (C-3), 78.5 (C-4), 112.8-152.0 (aromatic carbon), 168.0 (CO $\beta$-lactam); GC-MS m/z $=519\left[\mathrm{M}^{+}\right]$; Analysis calculated for $\mathrm{C}_{36} \mathrm{H}_{25} \mathrm{NO}_{3}: \mathrm{C}, 83.22: \mathrm{H}, 4.85 ; \mathrm{N}, 2.70 \%$. Found: C, 83.90; H, 4.80; N, 2.81\%. 
2-(Anthracen-9-yl)-1-phenylspiro[azetidine-3,9'-xanthen]-4-one (2d): Light yellow crystals from EtOAc (yield 63\%); Mp: 238-240 ${ }^{\circ} \mathrm{C} \quad$ (dec.); IR $\left(\mathrm{KBr}, \quad \mathrm{cm}^{-1}\right)$ : 1,758 (CO $\beta$-lactam); ${ }^{1} \mathrm{H}-\mathrm{NMR} \delta$ (ppm): 6.34 (H-4, s, 1H), 6.51-8.83 (ArH, m, 22H); ${ }^{13} \mathrm{C}-\mathrm{NMR} \delta(\mathrm{ppm}): 65.6$ (C-3), 75.4 (C-4), 115.9-152.0 (aromatic carbon), 167.5 (CO $\beta$-lactam); GC-MS m/z = $489\left[\mathrm{M}^{+}\right]$; Analysis calculated for $\mathrm{C}_{35} \mathrm{H}_{23} \mathrm{NO}_{2}$ : C, 85.87; H, 4.74; N, 2.86\%. Found: $\mathrm{C}, 85.87 ; \mathrm{H}, 4.74 ; \mathrm{N} 2.86 \%$.

2-(Anthracen-9-yl)-1-(4-chlorophenyl)spiro[azetidine-3,9'-xanthen]-4-one (2e): Yellow crystals from EtOAc (yield 70\%); Mp: $254-256{ }^{\circ} \mathrm{C}$ (dec.); IR (KBr, cm ${ }^{-1}$ ): 1,743 (CO $\beta$-lactam); ${ }^{1} \mathrm{H}-\mathrm{NMR} \delta$ (ppm): $6.30(\mathrm{H}-4, \mathrm{~s}, 1 \mathrm{H}), 6.52-9.06(\mathrm{ArH}, \mathrm{m}, 21 \mathrm{H}) ;{ }^{13} \mathrm{C}-\mathrm{NMR} \delta$ (ppm): 66.0 (C-3), 75.5 (C-4), 116.0-151.9 (aromatic carbon), 167.4 (CO $\beta$-lactam); GC-MS m/z $=524\left[\mathrm{M}^{+},{ }^{35} \mathrm{Cl}\right], 526\left[\mathrm{M}^{+},{ }^{37} \mathrm{Cl}\right]$; Analysis calculated for $\mathrm{C}_{35} \mathrm{H}_{22} \mathrm{ClNO}_{2}$ : C, 80.22; H, 4.23; N 2.67\%. Found: C, 80.28; H, 4.18; N, 2.53\%.

2-(Anthracen-9-yl)-1-(3-nitrophenyl)spiro[azetidine-3,9'-xanthen]-4-one (2f): Yellow crystals from EtOAc (yield 40\%); Mp: 212-214 ${ }^{\circ} \mathrm{C}$; IR $\left(\mathrm{KBr}, \mathrm{cm}^{-1}\right): 1,762$ (CO $\beta$-lactam); ${ }^{1} \mathrm{H}-\mathrm{NMR} \delta$ (ppm): $5.98(\mathrm{H}-4, \mathrm{~s}, 1 \mathrm{H}), 6.40-9.16(\mathrm{ArH}, \mathrm{m}, 21 \mathrm{H}) ;{ }^{13} \mathrm{C}-\mathrm{NMR} \delta(\mathrm{ppm}): 66.4$ (C-3), 75.8 (C-4), 112.4-152.1 (aromatic carbon), 169.2 (CO $\beta$-lactam); GC-MS m/z $=534\left[\mathrm{M}^{+}\right]$; Analysis calculated for $\mathrm{C}_{35} \mathrm{H}_{22} \mathrm{~N}_{2} \mathrm{O}_{4}$ : C, 78.64; H, 4.15; N, 5.24\%. Found: C, 78.63; H, 4.20; N, 5.32\%.

2-(Anthracen-9-yl)-1-(2-ethylphenyl)spiro[azetidine-3,9'-xanthen]-4-one (2g): White solid (yield 35\%); Mp: $208-210{ }^{\circ} \mathrm{C}$; IR (KBr, cm ${ }^{-1}$ ): 1,755 (CO $\beta$-lactam); ${ }^{1} \mathrm{H}-\mathrm{NMR} \delta$ (ppm): 1.14 (t, 3H, Me, $J=7.1), 4.00\left(\mathrm{CH}_{2}, \mathrm{q}, 2 \mathrm{H}, J=7.1\right), 4.92(\mathrm{~s}, 1 \mathrm{H}, \mathrm{H}-4), 6.30-8.74(\mathrm{ArH}, \mathrm{m}, 21 \mathrm{H}) ;{ }^{13} \mathrm{C}-\mathrm{NMR} \delta(\mathrm{ppm})$ : $15.1\left(\mathrm{CH}_{3}\right), 25.8\left(\mathrm{CH}_{2}\right), 64.5(\mathrm{C}-3), 75.1(\mathrm{C}-4), 115.9-152.2$ (aromatic carbon), 168.1 (CO $\beta$-lactam); GC-MS m/z $=517\left[\mathrm{M}^{+}\right]$; Analysis calculated for $\mathrm{C}_{37} \mathrm{H}_{27} \mathrm{NO}_{2}: \mathrm{C}, 85.85 ; \mathrm{H}, 5.26 ; \mathrm{N}, 2.71 \%$. Found: C, $85.83 ; \mathrm{H}, 5.30 ; \mathrm{N}, 2.76 \%$.

2-(Anthracen-9-yl)-1-(3-bromophenyl)spiro[azetidine-3,9'-xanthen]-4-one (2h): Yellow crystals from EtOAc (yield 55\%); Mp: 222-224 ${ }^{\circ} \mathrm{C}$; IR $\left(\mathrm{KBr}, \mathrm{cm}^{-1}\right)$ : 1,755 (CO $\beta$-lactam); ${ }^{1} \mathrm{H}-\mathrm{NMR} \delta$ (ppm): 6.18 $(\mathrm{H}-4, \mathrm{~s}, 1 \mathrm{H}), 6.23-8.65$ (ArH, m, 21H); ${ }^{13} \mathrm{C}-\mathrm{NMR} \delta$ (ppm): 66.0 (C-3), 75.6 (C-4), 115.7-151.9 (aromatic carbon), 167.7 (CO $\beta$-lactam); GC-MS m/z $=567\left[\mathrm{M}^{+},{ }^{80} \mathrm{Br}\right], 569\left[\mathrm{M}^{+},{ }^{82} \mathrm{Br}\right]$; Analysis calculated for $\mathrm{C}_{35} \mathrm{H}_{22} \mathrm{BrNO}_{2}$ : C, 73.95; H, 3.90; N, 2.46\%. Found: C, 73.90; H, 3.93; N, 2.51\%.

2-(Anthracen-9-yl)-1-(2,4-dimethoxyphenyl)spiro[azetidine-3,9'-xanthen]-4-one (2i): Light green crystals from EtOAc (yield 69\%); Mp: 180-182 ${ }^{\circ} \mathrm{C}$ (dec.); IR (KBr, $\mathrm{cm}^{-1}$ ): 1,739 (CO $\beta$-lactam); ${ }^{1} \mathrm{H}-\mathrm{NMR} \quad \delta$ (ppm): 2.91, 3.58 (2OMe, s, 6H) 6.16 (H-4, s, 1H), 6.17-8.18 (ArH, m, 20H); ${ }^{13} \mathrm{C}-\mathrm{NMR} \delta$ (ppm): 55.4, 55.5 (s, 6H, 2 OMe) 65.9 (C-3), 78.1 (C-4), 100.2-158.2 (aromatic carbon), 167.7 (CO $\beta$-lactam); GC-MS m/z = $549\left[\mathrm{M}^{+}\right]$; Analysis calculated for $\mathrm{C}_{37} \mathrm{H}_{27} \mathrm{NO}_{4}$ : C, 80.86; H, 4.95; N, 2.55\%. Found: C, 80.03; H, 4.98; N, 2.83\%.

2-(Anthracen-9-yl)-1-(3,4-dimethoxyphenyl)spiro[azetidine-3,9'-xanthen]-4-one (2j): Yellow solid (yield 91\%); Mp: $172-174{ }^{\circ} \mathrm{C}$; IR (KBr, cm ${ }^{-1}$ ): 1,743 (CO $\beta$-lactam); ${ }^{1} \mathrm{H}-\mathrm{NMR} \delta$ (ppm): 3.69, 3.97 (2OMe, s, 6H) $6.30(\mathrm{H}-4, \mathrm{~s}, 1 \mathrm{H}), 6.50-8.77$ (ArH, m, 20H); ${ }^{13} \mathrm{C}-\mathrm{NMR} \delta(\mathrm{ppm}): 55.9,56.1$ (2 OMe) 65.6 (C-3), 75.6 (C-4), 102.3-153.0 (aromatic carbon), 166.9 (CO $\beta$-lactam); GC-MS m/z = 549 [M ]; 
Analysis calculated for $\mathrm{C}_{37} \mathrm{H}_{27} \mathrm{NO}_{4}$ : C, 80.86; H, 4.95; N, 2.55\%. Found: C, 80.81; H, 4.97; $\mathrm{N}, 2.53 \%$.

2-(Anthracen-9-yl)-1-cyclohexylspiro[azetidine-3,9'-xanthen]-4-one (2k): Orange crystals from EtOAc (yield 69\%); Mp: $216-218{ }^{\circ} \mathrm{C}$; IR $\left(\mathrm{KBr}, \mathrm{cm}^{-1}\right)$ : 1,743 (CO $\beta$-lactam); ${ }^{1} \mathrm{H}-\mathrm{NMR} \delta$ (ppm): 1.53 , $1.75,1.95,2.22,2.65,3.81$ (cyclohexyl, m, 11H) $5.96(\mathrm{H}-4, \mathrm{~s}, 1 \mathrm{H}), 6.36-9.19$ (ArH, m, 17H); ${ }^{13} \mathrm{C}-\mathrm{NMR} \delta(\mathrm{ppm}): 24.5,25.5,30.0,31.0,56.7$ (cyclohexyl) 64.5 (C-3), 72.8 (C-4), 115.9-152.4 (aromatic carbon), 169.6 (CO $\beta$-lactam); GC-MS m/z = $495\left[\mathrm{M}^{+}\right]$; Analysis calculated for $\mathrm{C}_{35} \mathrm{H}_{29} \mathrm{NO}_{2}$ : C, 84.82; H, 5.90; N, 2.83\%. Found: C, 84.81; H, 5.92; N, 2.81\%.

2-(Anthracen-9-yl)-1-(naphthalen-1-yl)spiro[azetidine-3,9'-xanthen]-4-one (21): Orange crystals from EtOAc (yield 68\%); Mp: $184-186{ }^{\circ} \mathrm{C}$ (dec.); IR $\left(\mathrm{CHCl}_{3}, \mathrm{~cm}^{-1}\right)$ : 1,759 (CO $\beta$-lactam); ${ }^{1} \mathrm{H}-\mathrm{NMR} \delta$ (ppm): 6.45 (H-4, s, 1H), 6.61-9.8 (ArH, m, 24H); ${ }^{13} \mathrm{C}-\mathrm{NMR} \delta$ (ppme) 90.3 (C-3), 74.8 (C-4), 116.7-152.1 (aromatic carbon), 168.3 (CO $\beta$-lactam); GC-MS m/z $=539\left[\mathrm{M}^{+}\right]$; Analysis calculated for $\mathrm{C}_{39} \mathrm{H}_{25} \mathrm{NO}_{2}$ : C, 86.80; H, 4.67; N, 2.60\%. Found: C, 86.52; H, 4.63; N, 2.61\%.

1-(Naphthalen-1-yl)-2-(naphthalen-2-yl)spiro[azetidine-3,9'-xanthen]-4-one (2m): White solid (yield 50\%); Mp: $187-189{ }^{\circ} \mathrm{C}$; IR ( $\left.\mathrm{KBr}, \mathrm{cm}^{-1}\right)$ : 1,758 (CO $\beta$-lactam); ${ }^{1} \mathrm{H}-\mathrm{NMR} \delta$ (ppm) 5.73 (H-4, s, 1H), 6.50-9.00 (ArH, m, 22H); ${ }^{13} \mathrm{C}-\mathrm{NMR} \delta$ (ppm): 62.8 (C-3), 75.8 (C-4), 116.4-152.29 (aromatic carbon), 196.3 (CO $\beta$-lactam); GC-MS m/z = $489\left[\mathrm{M}^{+}\right]$; Analysis calculated for $\mathrm{C}_{35} \mathrm{H}_{23} \mathrm{NO}_{2}$ : C, 85.87; $\mathrm{H}, 4.74$; N, 2.86\%. Found: C, 85.67; H, 4.65; N, 2.79\%.

1-(4-Methoxyphenyl)-2-(naphthalen-2-yl)spiro[azetidine-3,9'-xanthen]-4-one (2n): Gray solid (yield 40\%); Mp: $184-186{ }^{\circ} \mathrm{C}$; IR $\left(\mathrm{CHCl}_{3}, \mathrm{~cm}^{-1}\right): 1,751$ (CO $\beta$-lactam); ${ }^{1} \mathrm{H}-\mathrm{NMR} \delta$ (ppm): 3.81 (OMe, s, $\left.3 \mathrm{H}\right)$ $5.21(\mathrm{H}-4, \mathrm{~s}, 1 \mathrm{H}), 6.64-8.33(\mathrm{ArH}, \mathrm{m}, 19 \mathrm{H}) ;{ }^{13} \mathrm{C}-\mathrm{NMR} \delta(\mathrm{ppm}): 55.5$ (OMe) $64.0(\mathrm{C}-3), 74.6(\mathrm{C}-4)$, 113.9-151.8 (aromatic carbon), 167.0 (CO $\beta$-lactam); GC-MS m/z $=469\left[\mathrm{M}^{+}\right]$; Analysis calculated for $\mathrm{C}_{32} \mathrm{H}_{23} \mathrm{NO}_{3}$ : C, 81.86; H, 4.94; N, 2.98\%. Found: C, 81.53; H, 4.98; N, 2.83\%.

\subsection{General Procedure for the Synthesis of Bis-Polycyclic Aromatic Spiro and Nonspiro- $\beta$-Lactams 4a-h and 6a-b}

A mixture of Schiff base $(1.00 \mathrm{mmol})$, triethylamine $(10.00 \mathrm{mmol}), 9 H$-xanthen-9-carboxylic acid or phenoxyacetic acid $(3.00 \mathrm{mmol})$ and tosyl chloride $(3.00 \mathrm{mmol})$ in dry $\mathrm{CH}_{2} \mathrm{Cl}_{2}(15 \mathrm{~mL})$ was stirred at room temperature for $24 \mathrm{~h}$. Then it was washed with $\mathrm{HCl} 1 \mathrm{~N}(20 \mathrm{~mL})$, saturated $\mathrm{NaHCO}_{3}(20 \mathrm{~mL})$ and brine $(20 \mathrm{~mL})$. The organic layer was dried $\left(\mathrm{Na}_{2} \mathrm{SO}_{4}\right)$, filtered and the solvent was evaporated to give the product as a crystal which was then purified by recrystallization from suitable organic solvents.

2-(Anthracen-9-yl)-1-(4-(4-(2-(anthracen-9-yl)-4-oxospiro[azetidine-3,9'-xanthene]-1-yl)benzyl)phenyl)spiro[azetidine-3,9'-xanthen]-4-one (4a): Orange solid (yield 92\%); Mp: $162-164{ }^{\circ} \mathrm{C}(\mathrm{dec}$.); IR $\left(\mathrm{KBr}, \mathrm{cm}^{-1}\right): 1,755$ (CO $\beta$-lactam); ${ }^{1} \mathrm{H}-\mathrm{NMR} \delta(\mathrm{ppm}): 3.76\left(\mathrm{CH}_{2}, \mathrm{~s}, 2 \mathrm{H}\right) 6.15(\mathrm{H}-4, \mathrm{~s}, 2 \mathrm{H}), 6.19-8.99$ (ArH, m, 42H); ${ }^{13} \mathrm{C}-\mathrm{NMR} \delta$ (ppm): $40.8\left(\mathrm{CH}_{2}\right) 65.7$ (C-3), 75.4 (C-4), 115.9-152.0 (aromatic carbon), 167.3 (CO $\beta$-lactam). Analysis calculated for $\mathrm{C}_{71} \mathrm{H}_{46} \mathrm{~N}_{2} \mathrm{O}_{4}: \mathrm{C}, 86.04 ; \mathrm{H}, 4.68 ; \mathrm{N}, 2.83 \%$. Found: C, 86.10; H, 4.71; N, 2.80\%. 
2-(Anthracen-9-yl)-1-(3-(3-(2-(anthracen-9-yl)-4-oxospiro[azetidine-3,9'-xanthene]-1-yl)benzyl) phenyl)spiro[azetidine-3,9'-xanthen]-4-one (4b): Orange solid (Yield 87\%); Mp: 220-222 ${ }^{\circ} \mathrm{C}$ (dec.); IR (KBr, cm $\left.{ }^{-1}\right): 1,758$ (CO $\beta$-lactam); ${ }^{1} \mathrm{H}-\mathrm{NMR} \delta$ (ppm): $3.78\left(\mathrm{CH}_{2}, \mathrm{~s}, 2 \mathrm{H},\right) 6.22(\mathrm{H}-4, \mathrm{~s}, 2 \mathrm{H}), 6.26-9.52$ (ArH, m, 42H); ${ }^{13} \mathrm{C}-\mathrm{NMR} \delta$ (ppm): $41.5\left(\mathrm{CH}_{2}\right) 59.6$ (C-3), 75.4 (C-4), 115.9-152.0 (aromatic carbon), 167.4 (CO $\beta$-lactam). Analysis calculated for $\mathrm{C}_{71} \mathrm{H}_{46} \mathrm{~N}_{2} \mathrm{O}_{4}: \mathrm{C}, 86.04 ; \mathrm{H}, 4.68 ; \mathrm{N}, 2.83 \%$. Found: C, 86.06; H, 4.70; N, 2.80\%.

2-(Anthracen-9-yl)-1-(4-(4-(2-(anthracen-9-yl)-4-oxospiro[azetidine-3,9'-xanthene]-1-yl)phenoxy) phenyl)spiro[azetidine-3,9'-xanthen]-4-one (4c): Yellow solid (Yield 75\%); Mp: $242-244{ }^{\circ} \mathrm{C}$ (dec.); IR $\left(\mathrm{KBr}, \mathrm{cm}^{-1}\right): 1,755$ (CO $\beta$-lactam); ${ }^{1} \mathrm{H}-\mathrm{NMR} \delta(\mathrm{ppm}): 6.20(\mathrm{H}-4, \mathrm{~s}, 2 \mathrm{H}), 6.22-8.68$ (ArH, m, 42H); ${ }^{13} \mathrm{C}-\mathrm{NMR} \delta(\mathrm{ppm}): 65.8$ (C-3), 75.5 (C-4), 115.9-153.9 (aromatic carbon), 167.0 (CO $\beta$-lactam). Analysis calculated for $\mathrm{C}_{70} \mathrm{H}_{44} \mathrm{~N}_{2} \mathrm{O}_{5}$ : C, 84.66; H, 4.47; N, 2.82\%. Found: C, 84.64; H, 4.48; N, 2.80\%.

2-(Anthracen-9-yl)-1-(3-(4-(2-(anthracen-9-yl)-4-oxospiro[azetidine-3,9'-xanthene]-1-yl)phenoxy) phenyl)spiro[azetidine-3,9'-xanthen]-4-one (4d): Orange solid (Yield 85\%); Mp: 140-142 ${ }^{\circ} \mathrm{C}$; IR (KBr, $\mathrm{cm}^{-1}$ ): 1,759 (CO $\beta$-lactam); ${ }^{1} \mathrm{H}-\mathrm{NMR} \delta$ (ppm): 6.15 (H-4, s, 2H), 6.40-8.79 (ArH, m, 42H). ${ }^{13} \mathrm{C}-\mathrm{NMR} \delta(\mathrm{ppm}): 65.8$ (C-3), 75.7 (C-4), 117.5-157.4 (aromatic carbon), 167.4 (CO $\beta$-lactam). Analysis calculated for $\mathrm{C}_{70} \mathrm{H}_{44} \mathrm{~N}_{2} \mathrm{O}_{5}$ : C, 84.66; H, 4.47; N, 2.82\%. Found: C, 84.63; H, 4.45; N, 2.81\%.

1,1'-(4,4'-Methylenebis(4,1-phenylene))bis(4-(anthracen-9-yl)-3-phenoxyazetidin-2-one) (4e): Yellow solid (Yield 80\%); Mp: $146-148{ }^{\circ} \mathrm{C}$; IR $\left(\mathrm{KBr}, \mathrm{cm}^{-1}\right)$ : 1,755 (CO $\beta$-lactam); ${ }^{1} \mathrm{H}-\mathrm{NMR} \delta$ (ppm): 3.42 $\left(\mathrm{CH}_{2}, \mathrm{~s}, 2 \mathrm{H}\right) 5.85(\mathrm{H}-4, \mathrm{~d}, 2 \mathrm{H}, J=2.8), 6.40(\mathrm{H}-3, \mathrm{~d}, 2 \mathrm{H}, J=2.8), 6.47-9.54(\mathrm{ArH}, \mathrm{m}, 36 \mathrm{H}) ;{ }^{13} \mathrm{C}-\mathrm{NMR}$ $\delta$ (ppm): $40.5\left(\mathrm{CH}_{2}\right) 62.9(\mathrm{C}-3), 59.1(\mathrm{C}-4), 115.6-157.1$ (aromatic carbon), 163.3 (CO $\beta$-lactam). Analysis calculated for $\mathrm{C}_{59} \mathrm{H}_{42} \mathrm{~N}_{2} \mathrm{O}_{4}$ : C, 84.06; H, 5.02; N, 3.32\%. Found: C, 84.10; H, 5.08; N, 3.35\%.

1,1'-(3,3'-Methylenebis(3,1-phenylene))bis(4-(anthracen-9-yl)-3-phenoxyazetidin-2-one) (4f): Orange solid (Yield 87\%); Mp: $118-120{ }^{\circ} \mathrm{C}$; IR $\left(\mathrm{KBr}, \mathrm{cm}^{-1}\right.$ ): 1,758 (CO $\beta$-lactam); ${ }^{1} \mathrm{H}-\mathrm{NMR} \delta$ (ppm): 3.47 $\left(\mathrm{CH}_{2}, \mathrm{~s}, 2 \mathrm{H}\right) 5.71(\mathrm{H}-4, \mathrm{~d}, 2 \mathrm{H}, J=3.6), 6.38(\mathrm{H}-3, \mathrm{~d}, 2 \mathrm{H}, J=3.6), 6.41-9.41(\mathrm{ArH}, \mathrm{m}, 36 \mathrm{H}) ;{ }^{13} \mathrm{C}-\mathrm{NMR}$ $\delta$ (ppm): $41.1\left(\mathrm{CH}_{2}\right) 82.6(\mathrm{C}-3), 60.6$ (C-4), 115.5-157.1 (aromatic carbon), 163.4 (CO $\beta$-lactam); Analysis calculated for $\mathrm{C}_{59} \mathrm{H}_{42} \mathrm{~N}_{2} \mathrm{O}_{4}$ : C, 84.06; H, 5.02; N, 3.32\%. Found: C, 84.08; H, 5.05; N, 3.30\%.

1,1'-(4,4'-Oxybis(4,1-phenylene))bis(4-(anthracen-9-yl)-3-phenoxyazetidin-2-one) (4g): Orange solid (Yield 90\%); Mp: $126-128{ }^{\circ} \mathrm{C}$; IR (KBr, cm $\left.{ }^{-1}\right): 1,755$ (CO $\beta$-lactam); ${ }^{1} \mathrm{H}-\mathrm{NMR} \delta$ (ppm): 5.71 (H-4, d, $2 \mathrm{H}, J=5.1), 6.20(\mathrm{H}-3, \mathrm{~d}, 2 \mathrm{H}, J=5.1), 6.36-8.78$ (m, ArH, 36H) ${ }^{13} \mathrm{C}-\mathrm{NMR} \delta(\mathrm{ppm}): 83.1(\mathrm{C}-3), 59.2$ (C-4), 115.6-133.0 (aromatic carbon), 167.7 (CO $\beta$-lactam). Analysis calculated for $\mathrm{C}_{58} \mathrm{H}_{40} \mathrm{~N}_{2} \mathrm{O}_{5}$ : C, 82.45; H, 4.77; N, 3.32\%. Found: C, 82.41; H, 4.79; N, 3.35\%.

4-(Anthracen-9-yl)-1-(3-(4-(2-(anthracen-9-yl)-4-oxo-3-phenoxyazetidin-yl)phenoxy)phenyl)-3-phen oxyazetidin-2-one (4h): Yellow solid (Yield 95\%); Mp: $118-120{ }^{\circ} \mathrm{C}$; IR $\left(\mathrm{KBr}, \mathrm{cm}^{-1}\right): 1,758(\mathrm{CO}$ $\beta$-lactam); ${ }^{1} \mathrm{H}-\mathrm{NMR} \delta$ (ppm): $5.75(\mathrm{H}-4, \mathrm{~d}, 2 \mathrm{H}, J=3.8), 6.25(\mathrm{H}-3, \mathrm{~d}, 2 \mathrm{H}, J=3.8), 6.42-8.79$ (ArH, m, $36 \mathrm{H}) ;{ }^{13} \mathrm{C}-\mathrm{NMR} \delta$ (ppm): 83.1 (C-3), 65.4 (C-4), 107.7-157.4 (aromatic carbon), 163.5 (CO $\beta$-lactam). Analysis calculated for $\mathrm{C}_{58} \mathrm{H}_{40} \mathrm{~N}_{2} \mathrm{O}_{5}$ : C, 82.45; H, 4.77; N, 3.32\%. Found: C, 82.43; H, 4.75; N, 3.30\%. 
1,1'-(1,4-Phenylene)bis(4-(anthracen-9-yl)-3-phenoxyazetidin-2-one) (6a): Gray solid (Yield 96\%); Mp: $178-180{ }^{\circ} \mathrm{C}$ (dec.); IR (KBr, cm $\left.{ }^{-1}\right): 1,751$ (CO $\beta$-lactam); ${ }^{1} \mathrm{H}-\mathrm{NMR} \delta$ (ppm): $5.58(\mathrm{H}-4, \mathrm{~d}, 2 \mathrm{H}$, $J=3.9), 6.30$ (H-3, d, 2H, $J=3.9), 6.33-8.82$ (ArH, m, 32H) ${ }^{13} \mathrm{C}-\mathrm{NMR} \delta$ (ppm): 83.0 (C-3), 59.1 (C4), 114.6-156.8 (aromatic carbon), 163.0 (CO $\beta$-lactam). Analysis calculated for $\mathrm{C}_{52} \mathrm{H}_{36} \mathrm{~N}_{2} \mathrm{O}_{4}$ : $\mathrm{C}$, 82.96; H, 4.82; N, 3.72\% Found: C, 82.95; H, 4.81; N, 3.70\%.

1,1'-(1,4-Phenylene)bis(2-(anthracen-9-yl)spiro[azetidine-3,9'-xanthen]-4-one) (6b): Orange solid (Yield 85\%); Mp: $186-188{ }^{\circ} \mathrm{C}$ (dec.); IR (KBr, cm $\left.{ }^{-1}\right): 1,751$ (CO $\beta$-lactam); ${ }^{1} \mathrm{H}-\mathrm{NMR} \delta$ (ppm): 6.27 $(\mathrm{H}-4, \mathrm{~s}, 2 \mathrm{H}), 6.30-8.99$ (ArH, m, 38H); ${ }^{13} \mathrm{C}-\mathrm{NMR} \delta$ (ppm): 59.7 (C-3), 75.2 (C-4), 115.9-152.0 (aromatic carbon), 167.0 (CO $\beta$-lactam). Analysis calculated for $\mathrm{C}_{64} \mathrm{H}_{40} \mathrm{~N}_{2} \mathrm{O}_{4}: \mathrm{C}, 85.31 ; \mathrm{H}, 4.47 ; \mathrm{N}$, $3.11 \%$. Found: C, 85.30; H, 4.45; N, 3.15\%.

\section{Conclusions}

This article describes for the first time the synthesis and characterization of some examples of mono-and bis-spiro- and nonspiro- $\beta$-lactams bearing a polycyclic aromatic moiety by reaction of polycyclic aromatic imines and two ketenes derived from $9 H$-xanthene-9-carboxylic acid and phenoxyacetic acid. These ketenes were prepared in situ with triethylamine and $p$-toluenesulfonyl chloride.

\section{Acknowledgements}

The authors thank the Shiraz University Research Council for financial support (Grant No. 88-GR$\mathrm{SC}-23)$.

\section{References}

1. Southgate, R. The synthesis of natural $\beta$-lactam antibiotics. Contemp. Org. Synth. 1994, 1, 417-431.

2. Broccolo, F.; Carnally, G.; Caltabiano, G.; Cocuzza, C.E.A.; Fortuna, C.; Galletti, G.; Giacomini, P.D.; Musumarra, G.; Musumeci, R.; Quitavalla, A. Design, synthesis, and biological evaluation of 4-alkyliden-beta lactams: new products with promising antibiotic activity against resistant bacteria. J. Med. Chem. 2006, 49, 2804-2811.

3. Alcaide, B.; Almendros, P.; Aragoncillo, C. $\beta$-Lactams: Versatile building blocks for the stereoselective synthesis of non- $\beta$-lactam products. Chem. Rev. 2007, 107, 4437-4492.

4. Alcaide, B.; Almendros, P. $\beta$-Lactams as versatile intermediates for the preparation of heterocycles of biological interest. Curr. Med. Chem. 2004, 11, 1921-1949.

5. Deshmukh, A.R.A.S.; Bhawal, B.M.; Krishnaswamy, D.; Govande, V.V.; Shinkre, B.A.; Jayanthi, A. Azetidin-2-ones, synthon for biologically important compounds. Curr. Med. Chem. 2004, 11, 1889-1920.

6. Alcaide, B.; Almendros, P. Selective bond cleavage of the $\beta$-lactam nucleus: Application in stereocontrolled synthesis. Synlett 2002, 381-393.

7. Palomo, C.; Aizpurua, J.M.; Ganboa, I.; Oiarbide, M. $\beta$-lactams as versatile intermediates in $\alpha$ and $\beta$-amino acid synthesis. Synlett 2001, 1813-1826. 
8. Ojima, I. The Organic Chemistry of B-Lactams; Georg, G.I., Ed.; VCH: New York, NY, USA, 1993; pp. 197-255.

9. Ojima, I. Recent advances in the $\beta$-lactam synthon method. Acc. Chem. Res. 1995, 28, 383-389.

10. O’Driscoll, M.; Greenhalgh, K.; Young, A.; Turos, E.; Dickey, S.; Lim, D.V. Studies on the antifungal properties of N-thiolated $\beta$-lactams. Bioorg. Med. Chem. 2008, 16, 7832-7837.

11. Turos, E.; Reddy, G.S.K.; Greenhalgh, K.; Ramaraju, P.; Abeylath, S.C.; Jang, S.; Dickey, S.; Lim, D.V. Penicillin-bound polyacrylate nanoparticles: restoring the activity of $\beta$-lactam antibiotics against MRSA. Bioorg. Med. Chem. Lett. 2007, 17, 3468-3472.

12. Dugar, S.; Yumibe, N.; Clader, J.W.; Vizziano, M.; Huie, K.; van Heek, M.; Compton, D.S.; Davis, H.R., Jr. Metabolism and structure activity data based drug design: discovery of (-) SCH 53079 an analog of the potent cholesterol absorption inhibitor (-) SCH 48461. Bioorg. Med. Chem. Lett. 1996, 6, 1271-1274.

13. Han, W.T.; Trehan, A.K.; Wright, J.J.K.; Federici, M.E.; Seiler, S.M.; Meanwell, N.A. Azetidin2-one derivatives as inhibitors of thrombin. Bioorg. Med. Chem. 1995, 3, 1123-1143.

14. Borthwick, A.D.; Weingarte, G.; Haley, T.M.; Tomaszewski, M.; Wang, W.; Hu, Z.; Bedard, J.; Jin, H.; Yuen, L.; Mansour, T.S. Design and synthesis of monocyclic $\beta$-lactams as mechanismbased inhibitors of human cytomegalovirus protease. Bioorg. Med. Chem. Lett. 1998, 8, 365-370.

15. Cainelli, G.; Galletti, P.; Garbisa, S.; Giacomini, D.; Sartor, L.; Quintavalla, A. 4-Alkylideneazetidin-2-ones: novel inhibitors of leukocyte elastase and gelatinase. Bioorg. Med. Chem. 2003, $11,5391-5399$.

16. Zhou, N.E.; Guo, D.; Thomas, G.; Reddy, A.V.N.; Kaleta, J.; Purisima, E.; Menard, R.; Micetich, R.G.; Singh, R. 3-Acylamino-azetidin-2-one as a novel class of cysteine proteases inhibitors. Bioorg. Med. Chem. Lett. 2003, 13, 139-141.

17. Kazi, A.; Hill, R.; Long, T.E.; Kuhn, D.J.; Turos, E.; Dou, Q.P. Novel N-thiolated $\beta$-lactam antibiotics selectively induce apoptosis in human tumor and transformed, but not normal or nontransformed, cells. Biochem. Pharmacol. 2004, 67, 365-374.

18. Alonso, E.; Lopez-Ortiz, F.; Del Pozo, C.; Peratta, E.; Macias, A.; Gonzalaz, J. Spiro $\beta$-lactams as $\beta$-turn mimetics. Design, synthesis, and NMR conformational analysis. J. Org. Chem. 2001, 66, 6333-6338.

19. Bittermann, H.; Gmeiner, P. Chirospecific synthesis of spirocyclic $\beta$-lactams and their characterization as potent type ii $\beta$-turn inducing peptide mimetics. J. Org. Chem. 2006, 71, 97-102.

20. Alonso, E.; Del Pozo, C.; Gonzalez, J. Synthesis of $\alpha, \alpha$-disubstituted $\beta$-amino esters and peptide derivatives. Synlett 2002, 69-72.

21. Pinder, J.L.; Weinreb, S.M. Preliminary feasibility studies on total synthesis of the unusual marine bryozoan alkaloids chartellamide A and B. Tetrahedron Lett. 2003, 44, 4141-4143.

22. Skiles, J.W.; McNeil, D. Spiro indolinone beta-lactams, inhibitors of poliovirus and rhinovirus 3C-proteinases. Tetrahedron Lett. 1990, 31, 7277-7280.

23. Jarrahpour, A.; Khalili, D. Synthesis of some mono- and bis-spiro- $\beta$-lactams of benzylisatin. Tetrahedron Lett. 2007, 48, 7140-7143.

24. Lin, X.; Weinreb, S.M. Model studies on total synthesis of the chartellines, spirocyclic $\beta$-lactam alkaloids from a marine bryozoan. Tetrahedron Lett. 2001, 42, 2631-2633. 
25. Anklam, S.; Liebscher, J. Synthesis of optically active spiro- $\beta$-lactams by cycloadditions to $\alpha$ alkylidene- $\beta$-lactams. Tetrahedron 1998, 54, 6369-6384.

26. Alcaide, B.; Almendros, P.; Del Campo, T.M.; Rodríguez-Acebes, R. Diversity-oriented preparation of enantiopure spirocyclic 2 -azetidinones from $\alpha$-oxo- $\beta$-lactams through barbier-type reactions followed by metal-catalyzed cyclizations. Adv. Synth. Catal. 2007, 349, 749-758.

27. Gareth, A.; Jonathan, C.; Stuart, D.H. Azabicyclic amino acids by stereoselective dearomatizing cyclization of the enolates of n-nicotinoyl glycine derivatives. Org. Lett. 2006, 8, 5325-5328.

28. Bhalla, A.; Venugopalan, P.; Bari, S.S. A new synthetic approach to novel spiro- $\beta$-lactams. Eur. J. Org. Chem. 2006, 4943-4950.

29. Zanobini, A.; Brandi, A.; De Meijere, A. A new three-component cascade reaction to yield 3spirocyclopropanated $\beta$-lactams. Eur. J. Org. Chem. 2006, 1251-1255.

30. Zanobini, A.; Gensini, M.; Magull, J.; Vidovic, D.; Kozhushkov, S.I.; Brandi, A.; De Meijere, A. A convenient new synthesis of 3 -substituted $\beta$-lactams formally derived from 1(aminomethyl)cyclopropanecarboxylic acids. Eur. J. Org. Chem. 2004, 4158-4166.

31. Alcaide, B.; Almendros, P.; Rodríguez-Acebes, R. Pd-Cu bimetallic catalyzed domino cyclization of $\alpha$-allenols followed by a coupling reaction: New sequence leading to functionalized spirolactams. Chem. Eur. J. 2005, 11, 5708-5712.

32. Bhalla, A.; Venugopalan, P.; Bhasin, K.K.; Bari, S.S. Seleno- $\beta$-lactams: synthesis of monocyclic and spirocyclic selenoazetidin-2-ones. Tetrahedron 2007, 63, 3195-3204.

33. Galliford, C.V.; Martenson, J.S.; Stern, C.; Scheidt, K.A. A highly diastereoselective, catalytic three-component assembly reaction for the synthesis of spiropyrrolidinyloxindoles. Chem. Commun. 2007, 631-633.

34. Sun, C.; Lin, X.; Weinreb, M. Explorations on the total synthesis of the unusual marine alkaloid chartelline A. J. Org. Chem. 2006, 71, 3159-3166.

35. Alcaide, B.; Almendros, P.; Rodr1'guez-Acebes, R. Efficient entry to diversely functionalized spirocyclic oxindoles from isatins through carbonyl-addition/cyclization reaction sequences. $J$. Org. Chem. 2006, 71, 2346-2351.

36. Cremonesi, G.; Dalla Croce, P.; La Rosa, C. Synthesis of imidazo[5,1-b]thiazoles or spiro- $\beta$ lactams by reaction of imines with mesoionic compounds or ketenes generated from $\mathrm{N}$-acylthiazolidine-2-carboxylic acids. Tetrahedron 2004, 60, 93-97.

37. Alcaide, B.; Almendros, P.; Del Campo, T.M.; Rodri'guez-Acebes, R. Metal-assisted synthesis of enantiopure spirocyclic $\beta$-lactams from azetidine-2,3-diones. Tetrahedron Lett. 2004, 45, 6429-6431.

38. Toshio, N.; Shigeo, K.; Minoru, I. Novel synthesis of bromoindolenine with spiro-blactam in chartelline. Synlett 2004, 2025-2027.

39. Arjona, O.; Csa'ky, A.G.; Murcia, M.C.; Plumet, J. The Staudinger reaction of imines derived from 7-oxanorbornenone: formation of spiranic oxazinone versus $\beta$-lactam rings. Tetrahedron Lett. 2002, 43, 6405-6408.

40. Alonso, E.; Del Pozo, C.; Gonzalez, J. Staudinger reactions of unsymmetrical cyclic ketenes: A synthetically useful approach to spiro $\beta$-lactams and derivatives. Reaction mechanism and theoretical studies. J. Chem. Soc. Perkin Trans. 1 2002, 571-576 
41. Cooper, I.R.; Grigg, R.; MacLachlan, W.S.; Thornton-Pett, M.; Sridharane, V. 3-Component palladium-indium mediated diastereoselective cascade allylation of imines with allenes and aryl iodides. Chem. Commun. 2002, 1372-1373.

42. Palomo, C.; Aizpurua, J.M.; Ganboa, I.; Oiarbide, M. Asymmetric synthesis of $\beta$-lactams through the staudinger reaction and their use as building blocks of natural and nonnatural products. Curr. Med. Chem. 2004, 11, 1837-1872.

43. Banik, I.; Becker, F.F.; Banik, B.K. Stereoselective synthesis of $\beta$-lactams with polyaromatic imines: Entry to new and novel anticancer agents. J. Med. Chem. 2003, 46, 12-15.

44. Banik, B.K.; Becker, F.F.; Banik, I. Synthesis of anticancer $\beta$-lactams: Mechanism of action. Bioorg. Med. Chem. 2004, 12, 2523-2528.

45. Becker, F.F.; Banik, B.K. Polycyclic aromatic compounds as anticancer agents: Synthesis and biological evaluation of some chrysene derivatives. Bioorg. Med. Chem. Lett. 1998, 8, 2877-2880.

46. Becker, F.F.; Banik, B.K. Unprecedented stereoselectivity in the Staudinger reaction with polycyclic aromatic imines. Tetrahedron lett. 2000, 41, 6551-6554.

47. Jarrahpour, A.; Alvand, P. Synthesis of some new sugar based 3,3-disubstituted monocyclic blactams by asymmetric [2+2] cycloaddition reactions. Iran J. Sci. Technol. Trans. A 2007, 31, $17-22$.

48. Jarrahpour, A.; Zarei, M. Synthesis of novel N-sulfonyl monocyclic $\beta$-lactams as potential antibacterial agents. Molecules 2006, 11, 49-58.

49. Jarrahpour, A.; Zarei, M. The Vilsmeier reagent as an efficient acid activator for the synthesis of $\beta$-lactams. Tetrahedron Lett. 2007, 48, 8712-8714.

50. Giuseppone, N.; Schmitt, J.L.; Schwartz, E.; Lehn, J.M. Scandium(III) catalysis of transimination reactions. Independent and constitutionally coupled reversible processes. J. Am. Chem. Soc. 2005, 127, 5528-5539.

51. Cabello, N.; Kizirian, J.C.; Alexakis, A. Enantioselective addition of aryllithium reagents to aromatic imines mediated by 1,2-diamine ligands. Tetrahedron Lett. 2004, 45, 4639-4642.

52. Jarrahpour, A.; Nazari, M.; Jalbout, A.F. Synthesis and physical characterization of 4-(anthracen10-yl)-1-cyclohexyl-3-phenoxyazetidin-2-one as a new Trans 2-azetidinone. Molbank 2007, M538.

53. He, L.; Jurs, P.C. Predicting the Genotoxicity of Polycyclic Aromatic Compounds from Molecular Structure with Different Classifiers. Chem. Res. Toxicol. 2003, 16, 1567-1580.

54. Akkurt, M.; Karaca, S.; Jarrahpour, A.; Ebrahimi, E.; Büyükgüngör, O. 4-(9-Anthr-yl)-1-(4methoxy-phen-yl)spiro[azetidin-3,9'-xanthen]-2-one. Acta Cryst. 2008, E64, 902-903. (Crystallographic data (excluding structure factors) for the structure in this Letter have been deposited with the Cambridge Crystallographic Data Centre as Supplementary Publication No. CCDC 688989 contains the supplementary crystallographic data for this paper. These data can be obtained free of charge via www.ccdc.cam.ac.uk/conts/retrieving.html or from the CCDC, 12 Union Road, Cambridge CB2 1EZ, UK; Fax: +44-1223-336033; E-Mail: deposit@ccdc.cam.ac.uk.)

55. Akkurt, M.; Yalcin, S.P.; Jarrahpour, A.; Ebrahimi, E.; Büyükgüngör, O. 4-(9-Anthryl)-1 (2methoxyphenyl)-spiro[azetidin-3,9'-xanthen]-2-one. Acta Cryst. 2009, E65, 0626-0627. 
56. Akkurt, M.; Celik, I.; Jarrahpour, A.; Ebrahimi, E.; Büyükgüngör, O. 4-(9-Anthryl)-1 phenylspiro[azetidine- 3,9'-xanthen]-2-one. Acta Cryst. 2009, E65, 501-502.

57. Akkurt, M.; Celik, I.; Jarrahpour, A.; Ebrahimi, E.; Büyükgüngör, O. 4-(9-Anthryl)-1-(3bromophenyl)spiro-[azetidine-3,9'-xanthen]-2-one. Acta Cryst. 2009, E65, o2522-o2523.

58. Akkurt, M.; Baktir, Z.; Jarrahpour, A.; Ebrahimi, E.; Büyükgüngör, O. 4-(9-Anthryl)-1-(2,4dimethoxyphenyl)-spiro[azetidine-3,9'-xanthen]-2-one. Acta Cryst. 2009, E65, o1623-o1624.

59. Akkurt, M.; Gencaslan, M.; Jarrahpour, A.; Ebrahimi, E.; Büyükgüngör, O. 4-(9-Anthryl)-1-(1naphthyl)spiro-[azetidine-3,9'-xanthen]-2-one $n$-hexane hemisolvate. Acta Cryst. 2008, E64, 2466-2467.

60. Amarego, W.L.; Chai, C.L.L. Purificatinon of Laboratory Chemicals 5th ed.; Elsevier: New York, NY, USA, 2003.

Sample Availability: Samples of the compounds $\mathbf{2 a}-\mathbf{n}, \mathbf{3 a}-\mathbf{d}, \mathbf{4 a}-\mathbf{h}$ and $\mathbf{6 a}-\mathbf{b}$ are available from authors.

(C) 2010 by the authors; licensee Molecular Diversity Preservation International, Basel, Switzerland. This article is an open-access article distributed under the terms and conditions of the Creative Commons Attribution license (http://creativecommons.org/licenses/by/3.0/). 